\title{
An analysis framework for identifying usability requirements in mobile application development
}

\begin{abstract}
People reasons to choose an application is for the functionalities offered. Though, the way in which the functions are performed will also have a significant impact to the user's choice. Software usability is one of the crucial factors that can influence user acceptance. It assesses the extent of which software facilitates users in utilizing the offered functions easily and effectively. Usability requirements are associated and complement the functional requirements. However, it is common that the usability requirements are captured at the design stage of software development due to its characteristic. Essentially, it should be identified at the earlier stage (i.e., requirements stage) to ensure the success of the software product. Therefore, we explore the potential benefits of Soft System Methodology (SSM) in identifying usability requirements at the requirements stage. The proposed SSM-based framework is applied in preliminary analysis, in which the results are useful to inform the next stage of the development process. The practical benefits of the proposed analysis framework are demonstrated in a case study. The framework would be beneficial particularly for mobile software developers in improving the quality of the mobile applications.
\end{abstract}

Keyword: Software requirements; Mobile application; Usability; Soft system methodology 